\title{
An in-vitro investigation on the effect of chilling and reheating different starchy meals on resistant starch content
}

\section{Abstract}

Food processing has been shown to influence starch digestibility, due to the formation of indigestible starches known as resistant starch (RS). RS has been shown to have similar health promoting properties to those of insoluble dietary fibre. These beneficial effects include lower glycaemic response, improved insulin sensitivity, enhanced overall gut health, and better plasma lipid profile. Additionally, the presence of other nutrients such as proteins or lipids may also impact the amount of RS formed.

The present work aimed to measure the effect of cooking and /or additional meal ingredients on the RS content of 5 different starchy meals, 3 made with pasta (one of which had cheese added) and 2 made with potato, using the Megazyme RS kit (K-RSTAR). The cooked meals were tested either freshly cooked, chilled overnight, or chilled overnight and reheated. Moreover, different amounts of fat were used in the test meals $(10 \mathrm{~g}$ fat per $100 \mathrm{~g}$ pasta, $30 \mathrm{~g}$ fat per $100 \mathrm{~g}$ pasta, $5 \mathrm{~g}$ fat per $100 \mathrm{~g}$ potato and $10 \mathrm{~g}$ fat per $100 \mathrm{~g}$ potato) in order to examine the effect of the amount of fat on RS formation. The total number of meals tested was 15 .

The amount of RS in the 2 pasta meals without cheese were $40 \%$ and $44 \%$ higher in the chilled and reheated meals compared to the same meals freshly cooked for the $10 \mathrm{~g}$ and $30 \mathrm{~g}$ of fat meals, respectively. The chilled overnight meals were not different from the freshly cooked meals for both fat amounts. Moreover, the potato meals showed a more substantial difference between the meals for the two fat amounts. The chilled and reheated potato meals were $73 \%$ and $85 \%$ higher in RS content than the freshly cooked identical meal, in the $5 \mathrm{~g}$ and $10 \mathrm{~g}$ of fat, respectively. Additionally, the chilled potato meals had higher RS contents compared to freshly cooked potato meals (68\% and $83 \%$, respectively). Furthermore, the presence of protein reduced the total amount of digested starch and RS in all pasta meals when compared to the meals without cheese. The findings of this in-vitro investigation suggests that chilling and reheating starchy meals significantly increases their content of RS. Future work will determine if these in vitro findings will translate to health benefits in vivo.

\section{Conflict of Interest}

There is no conflict of interest 